\title{
Consistent Steering System using SCTP for Bluetooth Scatternet Sensor Network
}

\author{
R. Dhaya $\cdot$ V. Sadasivam $\cdot$ R. Kanthavel
}

Received: 15 March 2012 / Accepted: 18 October 2012/Published online: 25 April 2013

(C) The Institution of Engineers (India) 2013

\begin{abstract}
Wireless communication is the best way to convey information from source to destination with flexibility and mobility and Bluetooth is the wireless technology suitable for short distance. On the other hand a wireless sensor network (WSN) consists of spatially distributed autonomous sensors to cooperatively monitor physical or environmental conditions, such as temperature, sound, vibration, pressure, motion or pollutants. Using Bluetooth piconet wireless technique in sensor nodes creates limitation in network depth and placement. The introduction of Scatternet solves the network restrictions with lack of reliability in data transmission. When the depth of the network increases, it results in more difficulties in routing. No authors so far focused on the reliability factors of Scatternet sensor network's routing. This paper illustrates the proposed system architecture and routing mechanism to increase the reliability. The another objective is to use reliable transport protocol that uses the multi-homing concept and supports multiple streams to prevent head-ofline blocking. The results show that the Scatternet sensor network has lower packet loss even in the congestive environment than the existing system suitable for all surveillance applications.
\end{abstract}

R. Dhaya ( $\square)$

Department of Information Technology, National Engineering

College, Kovilpatti 628503, Tamilnadu, India

e-mail: dhayavel@yahoo.co.in

V. Sadasivam

Department of Computer Science and Engineering,

Manonmaniam Sundaranar University, Tirunelveli 627012,

Tamilnadu, India

R. Kanthavel

Department of Computer Science and Engineering, Government College of Engineering, Tirunelveli 627007, Tamilnadu, India
Keywords Transport protocol $\cdot$ Scatternet . Sensor nodes $\cdot$ Bluetooth $\cdot$ Piconet

\section{Introduction}

Bluetooth is a wireless protocol for exchanging data over short distances from fixed and mobile devices, creating personal area networks (PANs). It can connect several devices and overcoming problems of synchronization. Bluetooth uses a radio technology called frequency-hopping spread spectrum [1]. Bluetooth provides a way to connect and exchange information between devices such as mobile phones, telephones, laptops, personal computers, printers, global positioning system (GPS) receivers and digital cameras. Bluetooth is generally considered as a promising short range wireless technology because of its inexpensive cost, low power and small size, and thus it has been gaining increasing interest from various industries [2].

Bluetooth protocols assume that a small number of units will participate in communications at any given time. These small groups are called piconets which consist of one master unit and up to seven active slave units. The master is the unit that initiates transmissions, and the slaves are the responding units [3]. As with piconets, where multiple Bluetooth devices are able to connect with each other in an ad-hoc manner is known as a Scatternet. A Scatternet is a type of ad-hoc computer network consisting of two or more piconets. A Scatternet is a number of interconnected piconets that supports communication between more than eight devices [4]. The device participating in both piconets is known as slave-bridge which can relay data between members of both ad-hoc networks. Using this approach, it is possible to join together numerous piconets into a large 
Scatternet, and to expand the physical size of the network beyond Bluetooth's limited range [5].

Bluetooth devices must have point-to-multipoint capability in order to engage in Scatternet communication, and several piconets can be connected to each other through one Scatternet [6]. Furthermore, a single Bluetooth device may participate as a slave in several piconets, but can only be a master in one piconet. On the other hand sensor nodes play an important role in all surveillance applications and the difficult thing is how different wireless modules are integrated in it. Moreover especially in sensor networks, it has many advantages to replace cables with wireless logical links [7]. Since in wireless sensor systems now are being applied by an international community for critical applications in security in a reliable way. Moreover head-of-line blocking a problem where sending independent messages over an order-preserving TCP connection causes delivery of messages sent later to be delayed within a receiver's transport layer buffers until an earlier lost message is retransmitted and arrives [8]. The main aim of the paper is to design a Sactternet sensor network in terms of reliability through routing with SCTP and almost nil-packet loss.

\section{Proposed Solution}

The proposed Scatternet consists of three phases namely setup, network configuration and routing. In setup phase, the participating nodes in the piconets of the Scatternet are arranged. In the network configuration stage, the inquiry procedure starts between nodes in the Scatternet. Finally in the routing stage, method of data flow is explained.

\section{Setup Phase}

A Bluetooth Scatternet sensor network comprising two piconets is established. Each piconet has one master and one slave sensor node. The slave called "slave-bridge" connects the two piconets. "Connects" here means that the slave-bridge acts as the medium through which data transmission takes place between the two masters. The two master nodes designed are to be multihomed. This suggests that, whenever a path breakage occurs, the proposed routing technique that automatically detects the path failure. Here all the Scatternet sensor nodes have been integrated via Bluetooth.

In Scatternet, sender node and receiver node possess addresses called IP1, IP2 and IP3, IP4. The purpose of having two addresses for providing the nodes to choose the best path during transmission in the congestive environment. The bridge node located in between the sender and receiver nodes is to relay the packets and taking responsibility to route the data.
Configuration Stage

In the Scatternet environments, nodes initially have no information about their surrounding environment or the nodes that operate within their range. There is no centralized instance to query about the environment. Therefore, a protocol must provide the facility for detecting nodes and should enable nodes to set-up connection. Two procedures used in the node discovery procedure are in terms of inquiry and page. In order to configure a connection, a node must detect what other devices are in range is the main goal of the inquiry procedure. The process is initiated by the network that wishes either to collect device information or to create a connection. The connection establishment is handled by the page process. The page process requires knowledge of the BD_ADDR of the device with which the connection is to be established. Furthermore, the device being paged must be in Page Scan sub-state, i.e. listening for page messages. At the end of the page process a connection has been set-up, the paging device becomes the master and the paged device becomes the slave. As with inquiry, a device typically entres page state periodically and a device that wishes to be able to connect to paging units that enters page scan in certain intervals. The BD_ADDR is nothing but a 48 bit length that is prescribed by the IEEE.

\section{Routing}

In the proposed Scatternet sensor network the routing is simple since every node has two addresses creating two paths, in which one path fails another comes in the routing scenario. The routing involves the following things:

- Two piconets, each with a master and a slave.

- First piconet consists of master 1 with two IP addresses: IP 1 and IP 2.

- Second piconet consists of master 2 with two IP addresses: IP 3 and IP 4.

- Slave bridge connects both the piconets.

- Primary path is chosen (if Node 1 fails)

Master $1 \rightarrow$ IP $1 \rightarrow$ Slave bridge $\rightarrow$ IP $3 \rightarrow$ Master 2

- Alternate path is chosen

Master $1 \rightarrow$ IP $2 \rightarrow$ Slave bridge $\rightarrow$ IP $4 \rightarrow$ Master 2

- Path for acknowledgement is Master $2 \rightarrow$ IP $4 \rightarrow$ Slave bridge $\rightarrow$ IP $2 \rightarrow$ Master

The proposed routing mechanism is illustrated as follows

Step 1: Initialize the Bluetooth Scatternet.

Step 2: Start data transferring through primary path.

Step 3: Start flowing heartbeat signals through alternate path. 
Step 4: If primary path fails-

- Stop data transferring through primary path.

- Search for an alternate path using heartbeat signals.

- Start data transferring through alternate path.

Step 5: Perform the data transmission through the alternate path until all data reaches successfully.

Step 6: Data transfer ends.

The protocol which have been used for the Scatternet sensor network formation is Stream Control Transmission Protocol (SCTP) which is an end-to-end transport protocol that providing reliable services and also supporting multihoming [9].The feature of SCTP is multihoming where a host with multiple points of attachment to the internet, for redundancy purposes, does not want to wait for a routing convergence to communicate critical messages to its peer communication endpoint [10]. For call control signalling, such delay is unacceptable when an alternate available path exists $[11,12]$. The path failure is found by the sender node due to the non receipt of acknowledge from the receiver node with in a stipulated time, which is normally called as round trip time (RTT). Since, the conventional routing methods, while using TCP,do not have the multihoming facility and only the data to be sent to the destination strictly in order, resulting congestion and path failure [12]. But the proposed method using SCTP increases reliability through multihoming facility.

\section{Experimental Results}

The experiments were done with the Network Simulator 2 (NS2) in Linux platform by taking different node levels of Scatternet network. By taking 3 nodes of 6, IP addresses for the first piconet of Scatternet the simulation has been started. At first Node 0 checks for other nodes in the Scatternet network, which are within its range. Node 1 , Node 2 and Node 3 are within the range of Node 0. So Node 0 can transmit data to these three neighbour nodes. In the same way every node in the network inquires about the neighbour nodes for the purpose of creating number of paths.

Secondly after initialisation, the data transfer starts from the sender to the receiver through the primary path. After data starts transferring through the primary path, heartbeat signals flow through the paths $3 \rightarrow 2$ and $2 \rightarrow 0$ automatically. Likewise the first piconet gets connected with the second one through bridge node.

Transmission times for different node levels for the primary path have been found from the Fig. 1 and it is identified that the transmission time gradually increases.

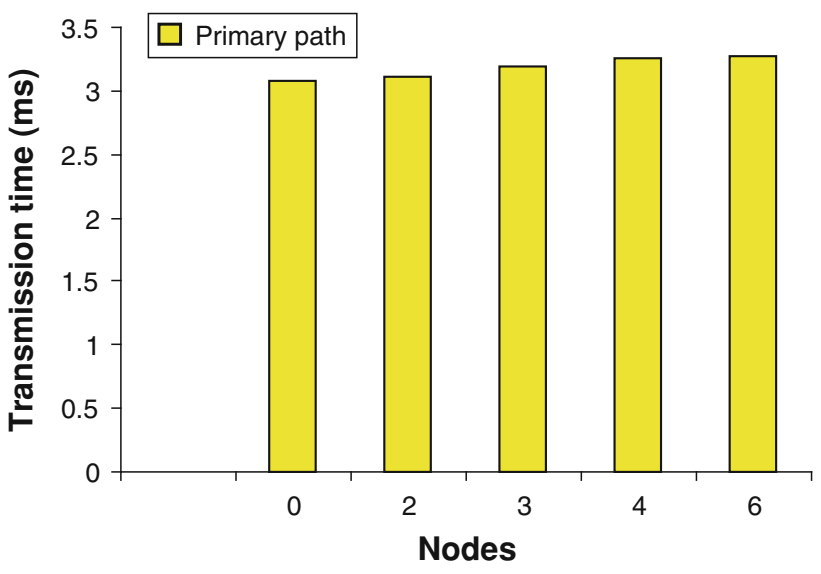

Fig. 1 Nodes against transmission time (primary path)

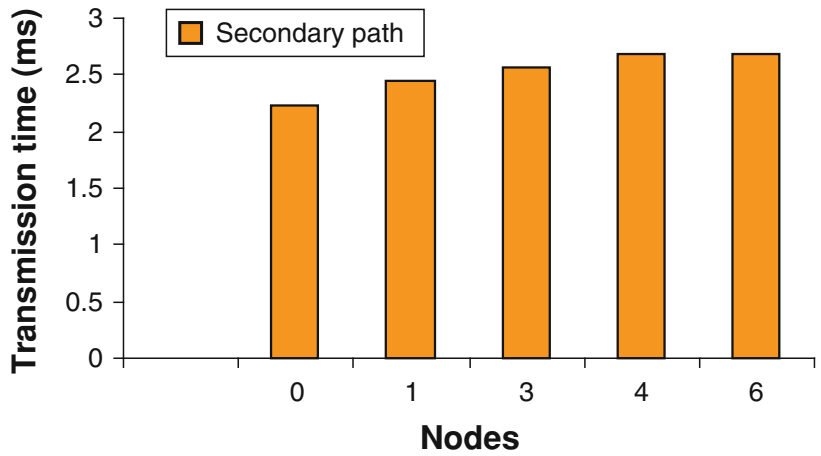

Fig. 2 Nodes against transmission time (secondary path)

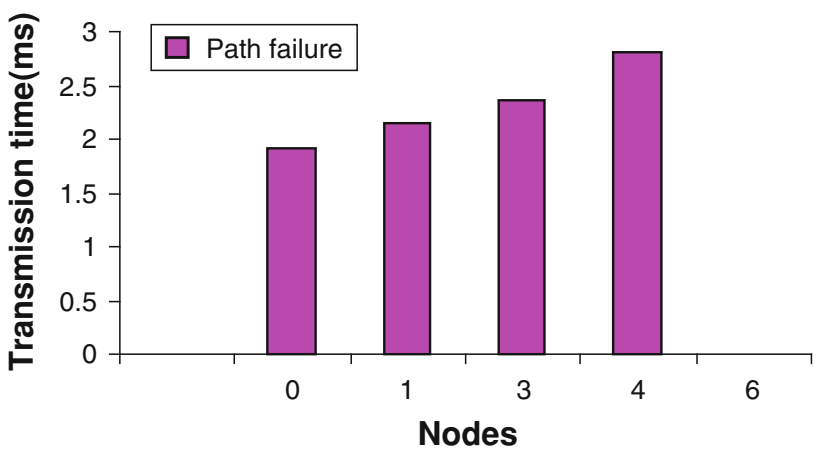

Fig. 3 Nodes against transmission time (primary path failure)

Likewise from Fig. 2 for the secondary path transmission time also gradually increases but which is less than the primary path.

The primary path failure has been deducted by the sender node after the fixed RTT.

Path failure for different node levels for the primary path and secondary path have been found and from the Figs. 3 and 4, it is identified that the transmission time varies with node levels and the transmission time is directionally proportional to the nodes in the network. 


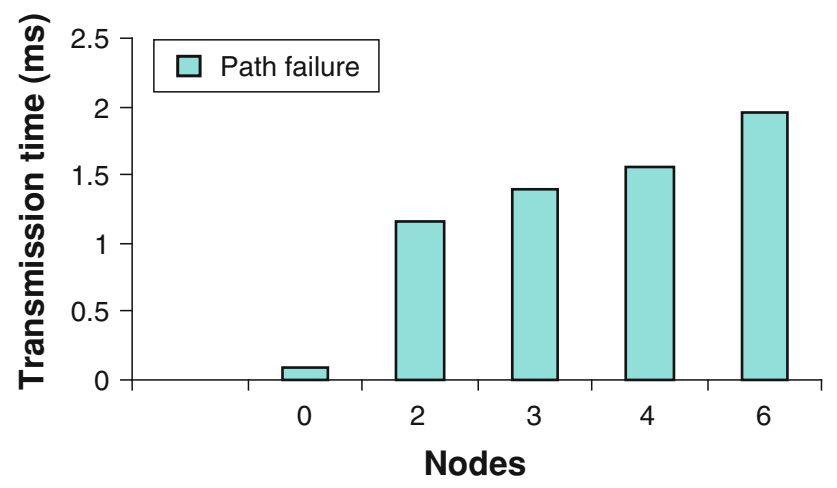

Fig. 4 Nodes against transmission time (secondary path failure)

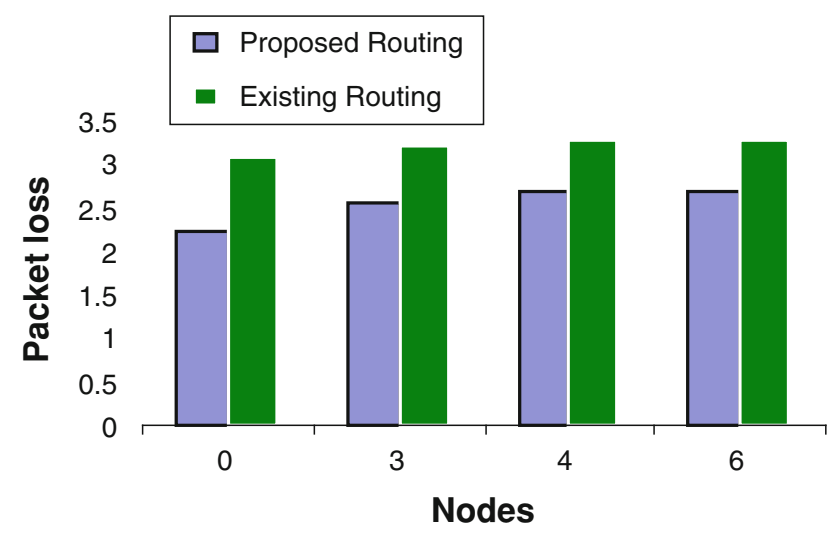

Fig. 5 Packet loss comparison between proposed routing with existing routing

Finally, the comparison between the proposed routing with existing routing [13] was done and it is concluded that the proposed one has lesser packet loss and transmission time as shown in Fig. 5 and it is also found that the reliability increases by using both the paths effectively. Thus the objective of increasing reliability in the way of reducing path failure using multihoming concept of SCTP has been achieved.

\section{Conclusion}

A Bluetooth Scatternet has been established. Multihoming facility of SCTP has been implemented in the established Bluetooth Scatternet. Hence congestion is avoided in the proposed system. From the experimental work the secondary path is more efficient than primary path. Secondary path transfers data, two times faster than the primary path. So even if primary path fails, data transmission through secondary path will be very efficient and reliable. SCTP thus increases the fault tolerance level during data transmission in Bluetooth Scatternets. In the proposed system every node can have two or more IP addresses. But it can be increased depending upon the need so that the number of paths for data flow could also be increased. Increased ACK traffic due to large amount of data transmission can be avoided by providing separate path also. So the packet loss has been highly reduced and but the same time reliability is not compromised.

\section{References}

1. A. Rodzevski, Creating a wireless sensor network using bluetooth technology. TR-TS-765. 1-4 (2004)

2. K. Scarfone, J. Padgette, Guide to bluetooth security. NIST Spec. Publ. 800-121. 1-43 (2008)

3. A. Edlund, Bluetooth wireless technology, business briefing. Wirel. Technolog. 28-31 (2005)

4. C. Petrioli, I. Chlamtac, S. Basagni, Configuring Bluestars: Multihop scatternet formation for bluetooth networks. IEEE Trans. Comput. 52(6), 779-790 (2003)

5. I. Howitt, Mutual interference between independent bluetooth piconets. IEEE Trans. Veh. Technol. 52(3), 708-718 (2003)

6. L. Jin, Y. Chang, Z. Li, A novel family of frequency hopping sequences for multi-hop bluetooth networks. IEEE Trans. Consum. Electron. 49(4), 1084-1089 (2003)

7. M.K. Afzal, A.U. Khan, A. Pescape, SCTP vs. TCP delay and packet loss, in IEEE Conference on multi topic, 1-5 (2007)

8. H. Chan, A. Perrig, Security and privacy in sensor networks. IEEE Comput. 36(10), 103-105 (2003)

9. G. Ye, M.J. Lee, T.N. Saadawi, Improving stream control transmission protocol performance over lossy links. IEEE J. Sel. Areas Commun. 22(4), 727-736 (2004)

10. P.D. Amer, A.L. Caro Jr., G.J. Heinz, J.R. Iyengar, S. Ladha, K.C Shah, SCTP: A proposed standard for robust internet data transport. Computer 36(11), 56-63 (2003)

11. J. Zou, M.U. Uyar, Fecko, M.A. Samtani, SF-SCTP: An extension of Stream Control Transmission Protocol to support QoS, in Proceedings of the 2006 IEEE International Conference on Networking, Sensing and Control, ICNSC 06, pp. 780-785 (2006)

12. R. Fracchia, C. Casetti, C.F. Chiasserini, M. Meo, A WiSE extension of SCTP for wireless networks. IEEE Int. Conf. Commun. 3, 1448-1453 (2005)

13. H. Kamal, B. Penoff, A. Wagner, SCTP versus TCP for MPI, in Proceedings of the ACM/IEEE SC 2005 Conference, on Supercomputing, pp. 30-35, 12-18 Nov 2005 